\title{
Nutcracker Syndrome, Vascular Imaging with Ultrasound - A Case Report
}

Svensson $\mathbf{C}^{1,2^{*}}$, Lassvik $\mathbf{C}^{1,2}$ and Zachrisson $\mathbf{H}^{1,2}$

${ }^{1}$ Department of Clinical Physiology University Hospital, Linköping, Sweden

${ }^{2}$ Department of Medicine and Health Science, Division of Clinical Physiology, Linköping University, Sweden

${ }^{*}$ Corresponding author: Svensson Christina, Department of Medicine and Health Science, Division of Clinical Physiology, Linköping University, Sweden, Tel: +46-10-1030000; E-mail: Christina.Svensson@regionostergotland.se

Received date: February 08, 2018; Accepted date: March 05, 2018; Published date: March 12, 2018

Copyright: () 2018 Svensson C, et al. This is an open-access article distributed under the terms of the Creative Commons Attribution License, which permits unrestricted use, distribution, and reproduction in any medium, provided the original author and source are credited.

\begin{abstract}
Nutcracker Syndrome (NCS) or Left Renal Vein (LRV) entrapment is rare and may be caused by compression of the vein between the aorta and the Superior Mesenteric Artery (SMA). LRV hypertension may lead to varicosities. The syndrome is characterized by a complex of ymptoms with substantial variations, the diagnosis is difficult and therefor often delayed. The diagnosis may be settled by many imaging methods, such as renal angiography, retrograde phlebography, Computed Tomography (CT), Magnetic Resonance Imaging (MRI) and Doppler Ultrasonography (DUS). The degree of stenosis may be detected measuring Doppler flow velocities by DUS.

A 50-year old female with Crohn's disease treated by ileocecal resection, performed 20 years ago, and symptomatic epilepsy treated by a vagal stimulator, had newly added symptoms of abdominal pain, weight loss, dizziness, irregular bowel movements, and increasing fatigue. Blood parameters and physical examination were normal. CT showed no signs of active Crohn's disease. The left renal veins and the left ovarian vein were dilated and the aorto-mesenteric angle was only 22 degrees.

DUS (Siemens S2000, 6 and $9 \mathrm{MHz}$ transducers) performed one month later confirmed the findings of the CT with typical findings of NCS. DUS will play a crucial role in Nutcracker syndrome considering flow velocity, diameter measurement, anatomy and aorto-mesenteric angle. DUS is a non-invasive, cheap modality that gives a very good resolution that can define vessel walls and evaluate flow velocity conditions. This patient had mild symptoms that led to a conservative treatment whereas epilepsy and Crohn's disease was the main problem. If severe symptoms occur different kinds of treatments are available, such as stenting of LRV, open surgical interventions, ablation of collateral pelvic veins and coil embolization.
\end{abstract}

Keywords: Vascular; Nutcracker syndrome; Anatomy; Cardiovascular

\section{Introduction}

Nutcracker syndrome (NCS), Nutcracker Phenomenon (NCP) or left renal vein entrapment syndrome is a condition that refers to compression of the left renal vein between the aorta and the origin of the SMA (Figure 1). The outflow from the LRV into inferior vena cava will be affected which results in renal vein hypertension leading to the development of collateral veins and perirenal varicosities. The syndrome is characterized by a complex set of symptoms with substantial variations, the diagnosis is difficult and therefore often delayed. The type of nutcracker syndrome in this case report is known as anterior nutcracker $[1,2]$. Other types of NCP are a combination of anterior nutcracker and compression of the third portion of duodenum that courses in front of the LRV between the aorta and the SMA. This condition is known as Willkie syndrome or superior mesenteric artery syndrome. There is also a posterior nutcracker syndrome that origins from a compression of the retroaortic vein between the aorta and the vertebral body. The space between aorta and superior mesenteric artery is often $4-5 \mathrm{~mm}[2,3]$, the angle between the vessels is maintained by retroperitoneal fat and the third part of duodenum with a normal angle of $38-56^{\circ}$. A thin body with decreased visceral fat, left renal ptosis with stretching of the LRV or abnormal angle in the origin of superior mesenteric artery from the aorta could be theories of cause.
Compression can also have other causes such as tumors, fibrolymphatic tissue, lymphadenopathy and overarching testicular artery [2].

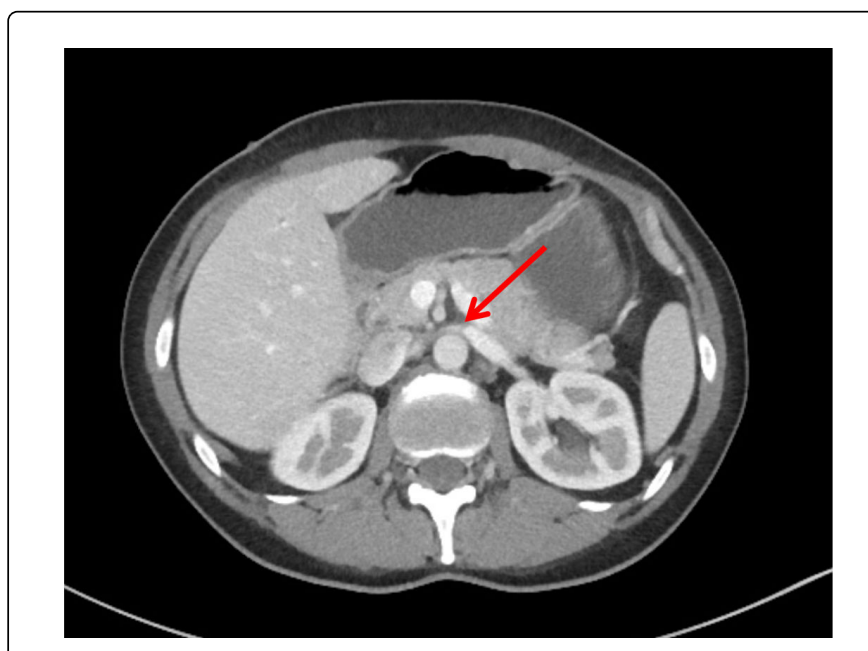

Figure 1: CT-scan of abdomen demonstrating impingement (red arrow) of the LRV by the SMA. CT Computed tomography, LRV Left renal vein, SMA, Superior mesenteric artery. 
Citation: Svensson C, Lassvik C, Zachrisson H (2018) Nutcracker Syndrome, Vascular Imaging with Ultrasound - A Case Report. Cardiovasc Pharm Open Access 7: 235. doi:10.4172/2329-6607.1000235

Page 2 of 3

Hematuria is the most commonly symptom, the cause of this is rupture of thin wall varices due to elevated venous pressure. These can later lead to anemia. Pain is also a common symptom and is characterized by abdominal or flank pain, the pain is worsening in standing or sitting position and exercise due to visceral proptosis.

NCS can also lead to severe pelvic congestion. Fatigue symptoms have also been associated with NCS. Varicocele is another symptom, most often occurring on the left side. NCS can also lead to atypical varicose veins because of collateral development, and venous insufficiency of the lower limb [2,3].

For diagnosis, after a clinical examination and blood examination, urinalysis and different renal studies there are multiple imaging modalities that can be used, such as renal angiography, CT, Magnetic resonance imaging (MRI) and Doppler ultrasonography (DUS) [2-4].

DUS is a noninvasive cheap method and could be the next step if a NCS is suspected on CT or MRI. With DUS it is possible to measure flow velocities and diameters in LRV, a ratio of both velocity and diameter between the hilar and narrowed part of the vessel $>5$ are used as NSV-criteria (Cho et al.). A flat angle (below 38-56 ${ }^{\circ}$ ) between aorta and SMA is also diagnostic criteria [2].

Conservative treatment is the first choice of treatment. If severe and persistent symptoms such as severe pain, severe hematuria, renal insufficiency and conservative treatment failure intervention such as LRV-stent, LRV-graft, LRV-transposition and coil embolization of varicose veins could be needed $[2,3,5]$.

\section{Case History}

A 50-year old female was referred to the department of clinical physiology due to a suspected NCS at a CT-scan. The patient had a history of Crohn's disease since approximately twenty-five years, an ileocecal resection was done twenty years ago.

Two colposcopies that showed stenotic ileocolic anastomosis were conducted in the previous six months. No subileus symptoms. She also had severe epilepsy (no grand mal) and a vagus stimulator for eleven years, MRI could therefore not be performed.

She is now presented with a newly added symptom of abdominal pain, weight loss, fatigue, dizziness and irregular bowel movement. On physical examination, abdominal palpation was without remarks. Laboratory tests showed slightly increased calprotectin, normal hemoglobin level and no hematuria.

The actual CT-scan with intravenous contrast showed a significant ileocolic stenosis, there were no signs of inflammatory intestinal activity, there were no edema, no pathological contrast enhancement in the intestinal walls and no dilated vasa recta.

As an incidental finding a NCS was suspected, a flat aortomesenteric angle of $22^{\circ}$, a partial compressed LRV (within SMA origin) and a dilated left ovarian vein were seen (Figure 2). Because of these findings a flow velocity measurement with DUS was recommended.

DUS were performed in the department of clinical physiology one month later and confirmed the findings of the CT. The patient was examined in a supine position after four hours of fasting, diameters and flow velocities were measured with Siemens S 2000, a 6C2- and 9L4-transducer were used.

A typical image with dilated distal LRV, narrowed LRV within the origin of SMA and affected flow velocities in the different parts of LRV.
A wall thrombosis was also seen just distal of the narrowed part of the LRV (Figures 3 and 4). A flat aortomesenteric angle was confirmed (Figure 5).

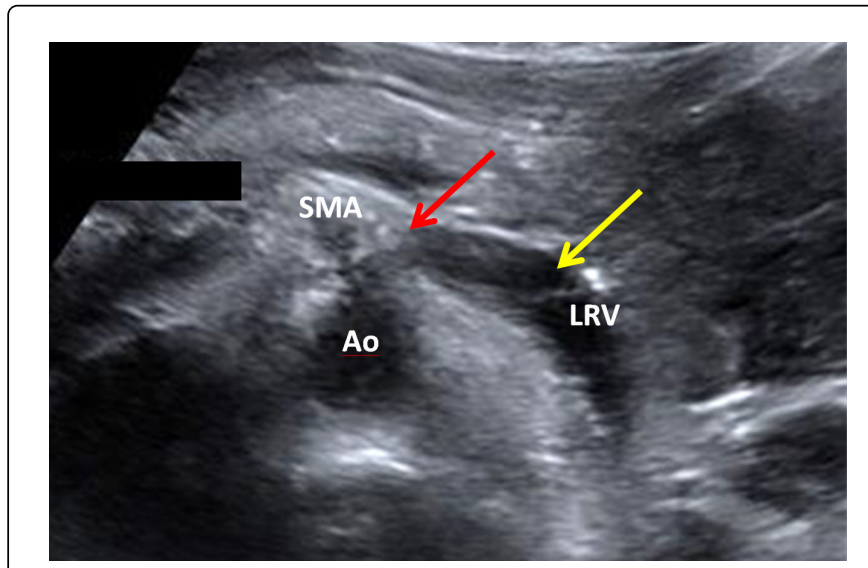

Figure 2: DUS of LRV with compression in the origin of SMA (red arrow). A wall thrombosis is also seen (yellow arrow). DUS Duplex ultrasound, Ao Aorta, LRV Left renal vein, SMA Superior mesenteric artery.

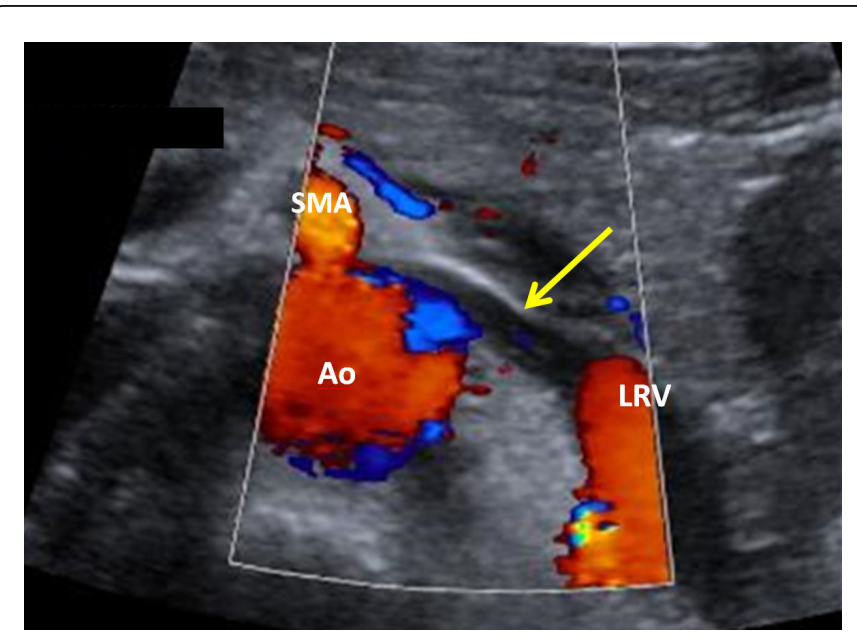

Figure 3: Colour Doppler of flow conditions in LRV. No detectable flow in the thrombotic part of the vein (yellow arrow). Ao Aorta, LRV Left renal vein, SMA superior mesenteric artery.

When measuring diameter in the left renal vein there were a huge difference between the distal and narrowed part. Calculating ratio resulted in five. Flow velocity measurments in LRV also showed clear differences with a ratio of seven between the distal and narrowed part.

Since the patient had no severe symptoms she had conservative treatment. The patient's major problem is the epilepsy and the symptoms of Crohn's disease which is treated with antiepileptic drugs respectively nutrition treatment. Antiplatelet aggregation treatment is also added. 


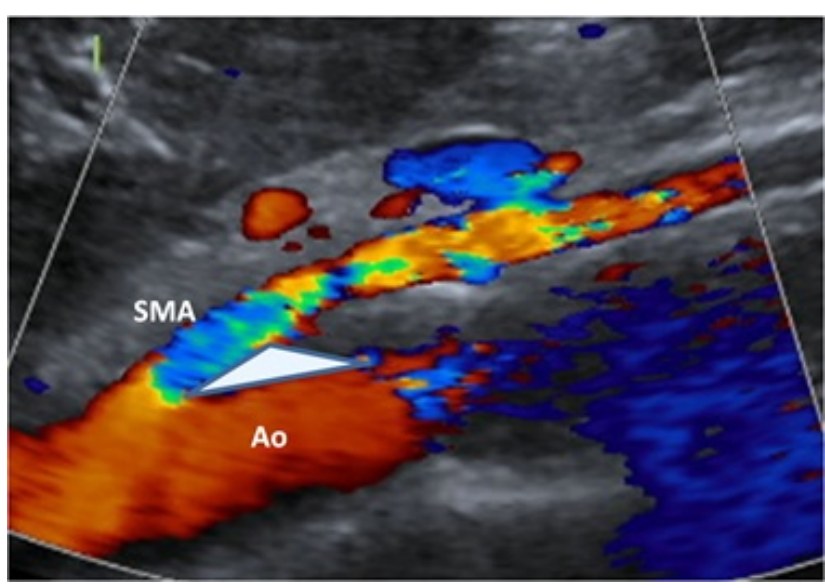

Figure 4: A flat aortomesenteric angle (white triangle). Ao Aorta, SMA superior mesenteric artery.

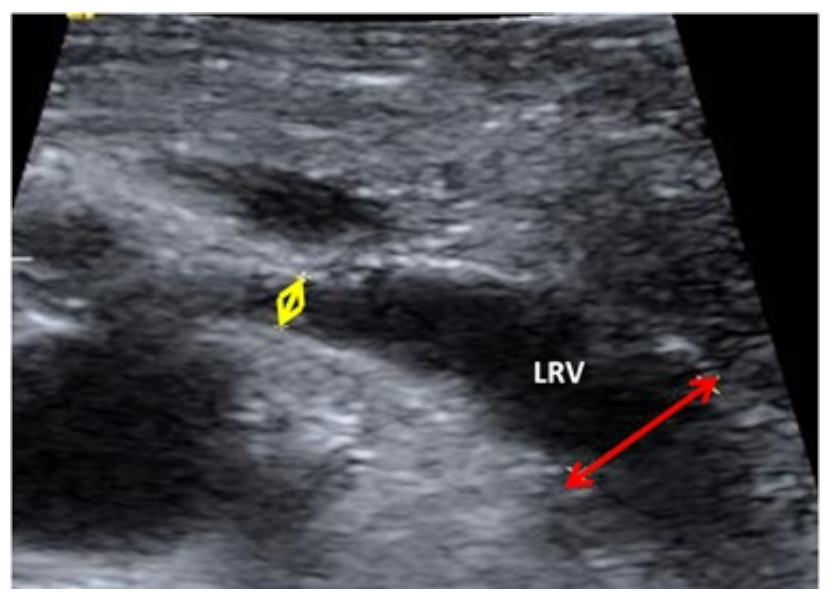

Figure 5: Diameter measurement of LRV, distal (red mark) and narrowed part (yellow mark). LRV Left renal vein.

\section{Discussion}

The first clinical report of NCS was by El-Sadr and Mina in 1950 (Ahmed et al.). The prevalence of NCS is unknown, it may be slightly higher in females and it is seen in all ages [2].

NCS is an uncommon, often underdiagnosed disease that can develop at any age and may be encountered by physicians in many disciplines. Suspicion for NSC should come to mind if the patient's symptoms are hematuria, abdominal flank pain, pelvic varicosities and varicoceles. These are the most common clinical signs of the disease. The term NCS is suggested to be reserved for patients with characteristic clinical symptoms in combination with nutcracker morphologic features. The investigation chain includes physical examination, laboratory testing, cystoscopy, ureteroscopy and different kinds of imaging modalities [2]. In combination with CT and MRI, DUS will play a crucial role considering flow velocity, diameter measurement, and anatomy and aortomesenteric angle.

DUS is a noninvasive, cheap modality that gives very good resolution that can define vessel walls and evaluate flow velocity conditions. Because of this DUS should be the first assessment if NCS is suspected clinically. CT-angiography is often a choice in the diagnosis of renal vein hypertension because of the possibility to do pressure measurements during the examination. MRI is also a choice in imaging, with the advantage of being radiation free. The possibility of multi-planer images that shows good anatomic images of surrounding tissues in both methods can also exclude other causes of pain and hematuria.

In this presented case a combination of physical investigation, CT and DUS confirmed the NSC diagnosis. This patient had mild symptoms that led to a conservative treatment, her epilepsy and Crohn 's disease was the main problem. If severe symptoms occur different kinds of treatments are available, such as stenting of LRV, open surgical interventions, ablation of collateral pelvic veins and coil embolization.

Conservative treatment is recommended when mild symptoms or mild hematuria is present. Considering orthostatic proteinuria angiotensin inhibitors might be a treatment option. Interventions should be considered when persisting or severe symptoms occur such as severe pain, significant hematuria, renal insufficiency or nonresponding to conservative treatment.

If severe symptoms occur different kinds of treatments are available, such as stenting of LRV, open surgical interventions, ablation of collateral pelvic veins and coil embolization.

Most interventions aim to decrease left renal vein hypertension, but others are aimed to treat pelvic venous reflux.

It has to be considered that complications might occur after intervention including migration of the stent and rarely thrombosis. Anticoagulation is recommended for at least 2-3 months after the procedure. Stent restenosis, deformities, and erosions at the placement site are other potential risks after intervention.

Both stenting and open surgical interventions may relieve symptoms however, selection criteria are not well-defined and clinical and diagnostic follow up may be necessary.

\section{References}

1. Hanna HE (1997) Nutcracker syndrome: an underdiagnosed cause for hematuria. SDJ Med 50: 429-436.

2. Kurklinsky AK, Rooke TW (2010) Nutcracker phenomenon and nutcracker syndrome. Mayo Clin Proc 85: 552-559.

3. Ahmed K (2006) Current trends in the diagnosis and management of renal nutcracker syndrome: a review. Eur J Vasc Endovasc Surg 31: $410-416$.

4. Cho BS (2001) Diagnosis of nut-cracker phenomenon using renal Doppler ultrasound in orthostatic proteinuria. Nephrol Dial Transplant 16: $1620-1625$.

5. Barsoum MK (2008) Patient with both Wilkie syndrome and nutcracker syndrome. Vasc Med 13: 247-250. 